childhood sexual abuse is a significant factor in the histories of some adults presenting with depressive syndromes.

Second, this period was also a time when attention-deficit hyperactivity disorder (ADHD) was not recognised and hyperkinetic disorder was only rarely diagnosed. Some of the young people, especially those in the comorbid conduct disorder/ major depressive disorder group, may have had undiagnosed and untreated ADHD. Certainly this was long before the use of psychostimulants on a wider basis in the UK and it is possible that some of these young people untreated may have been more vulnerable to development of depressive syndromes because of untreated attentional and other behavioural problems impacting on their self-esteem.

Third, although antidepressants were in use by child and adolescent psychiatrists when the diagnosis was major depressive disorder, they may not always have been used in young people with major depressive disorder with comorbid conduct disorder because of the risks of overdose in such a population. Tricyclic antidepressants were the predominant antidepressants used at that time in this population. With the advent of selective serotonin reuptake inhibitors, child and adolescent psychiatrists probably began prescribing more antidepressants in the comorbid conduct disorder/major depressive group because of the lower risk of serious harm in overdose. This raises the possibility that more effective treatment of these young people might also have an impact on their outcomes in adult life.

Fombonne, E., Wostear, G., Cooper, V., et al (200I) The Maudsley long-term follow-up of child and adolescent depression. I. Psychiatric outcomes in adulthood. British Journal of Psychiatry, 179, 210-217.

J. Hynes, N. McCune Child and Family Clinic, Bocombra Lodge, 2 Old Lurgan Road, Portadown BT63 5SG, UK

Author's reply: The comments of Hynes \& McCune raise pertinent questions. As they point out, it is possible that sexual abuse in childhood might have influenced the onset of juvenile depression, and also the likelihood of adult depression recurrence in our sample. In this study, we have collected data on sexual abuse, using both a review of medical charts at the time of Maudsley attendance and from adult interviews based on the Childhood Experience of Care and Abuse (CECA) measure. The effect of sexual abuse in childhood on patterns of adult depression recurrence will be investigated in the next analyses of this data-set, with particular attention given to differential risk processes according to childhood comorbidity.

Regarding comorbid ADHD as a risk factor for adolescent depression, particularly in the depression group with comorbid conduct disorder, we found a significantly increased rate of ADHD in the comorbid group, as we reported (Fombonne et al, $2001 a$, Table 2). Yet, it is plausible that the rate of ADHD in this sample was underestimated as many cases were ascertained before ADHD or hyperkinetic disorders were fully recognised as valid diagnostic entities. Nevertheless, our findings suggest that it is possible that ADHD might have been implicated in the development of conduct symptoms in the comorbid group although, because of the small sample size and likely underestimation of ADHD in that group, we cannot test for the specific contribution of (untreated) ADHD in the onset and recurrence of depression.

We had provided explicit data on the use of tricyclic antidepressant drugs during childhood years and found that the rate of prescriptions of these drugs was significantly higher in the non-comorbid group than do the comorbid group $(48.4 \% v$. $30.2 \%, P=0.032$; see Fombonne et al, 2001a). Most of these prescriptions were for amitriptyline and relied on dosages much lower than those considered appropriate by today's standards. Although the rate of antidepressant use was lower in the comorbid group, antidepressants were nevertheless often prescribed in that group too. Obviously, we could not assess whether or not use of tricyclic medications in that sample influenced long-term outcomes, since our study relied on an observational design. The interesting aspect of these data was to point to the frequent use by practising child psychiatrists of antidepressant drugs (irrespective of their known efficacy) in this sample of youths with depression assessed in the 1970s at a time when child and adolescent depression was largely ignored in professional training and in the literature. Furthermore, the data indirectly validated our diagnostic procedures.

Most of the comments by Hynes \& McCune raise questions about the mechanisms underlying recurrence of depression in adulthood following a first episode in childhood or adolescence. The findings of our study (Fombonne et al, $2001 a, b)$ indicated that relapse rates were similar, irrespective of the presence of comorbid conduct disorder in childhood. This result is important in its own right as it refutes previous hypotheses that depression, when occurring in the context of conduct disturbances, reflected mostly local psychosocial circumstances and was not associated with long-term heightened risk of affective disorders in adulthood.

This study was designed to assess mechanisms underlying recurrence of depression in adult life and further reports will address the role of early childhood experiences (such as sexual abuse), life events, family history and individual psychological characteristics on the patterns of adult depressive recurrence. It could well be that, in spite of having similar rates of relapse in adulthood, the mechanisms of depressive recurrence differ for the two groups included in this study, according to childhood comorbidity.

Fombonne, E., Wostear, G., Cooper, V., et al (200la)

The Maudsley long-term follow-up of child and adolescent depression. I. Psychiatric outcomes in adulthood. British Journal of Psychiatry, 179, 210-217.

_, _ _ _ , et al (200lb) The Maudsley long-term follow-up of child and adolescent depression. 2 Suicidality, criminality and social dysfunction in adulthood. British Journal of Psychiatry, 179, 218-223.

E. Fombonne McGill University, Department of Psychiatry, Montreal Children's Hospital, 4018 Ste-Catherine West, Montreal H3Z IP2, Canada

\section{Genetics of Down's syndrome and Alzheimer's disease}

In an extremely interesting article which touched upon early-onset dementia I feel that Dr Holmes (2002) failed to mention Down's syndrome as being a particular risk factor for the development of early-onset Alzheimer's disease because of triplication of the amyloid precursor gene. It is well known that almost all adults over the age of 40 years with Down's syndrome display Alzheimer's neuropathology (Mann, 1988) and the prevalence of dementia in people with Down's syndrome is $0-4 \%$ under the age of 30 years rising to $29-75 \%$ at $60-65$ years of age, which falls under the category of early-onset Alzheimer's disease (Zigman 
et al, 1997). Studies have shown that the prevalence of Alzheimer's disease in those with learning disability, especially Down's syndrome, is higher than in those with no learning disability (Patel et al, 1993).

The occurrence of Alzheimer-like neuropathology in Down's syndrome suggests that the genetic defect for familial Alzheimer's disease might reside on chromosome 21, which was, therefore, the first of the 22 autosomes to be tested using a genetic linkage strategy (McGuffin et al, 1994).

Dr Holmes's article was published among papers with the overall topic of old age psychiatry; early-onset Alzheimer's disease tends to fall within the remit of old age psychiatry except in those with Down's syndrome, who remain within learning disability services. From all the information I have gathered on Alzheimer's disease I have assumed that the clearest evidence for a genetic contribution to the aetiology of Alzheimer's disease is its association with Down's syndrome, which surely deserves a mention when discussing this specific area.

Holmes, C. (2002) Genotype and phenotype in Alzheimer's disease. British Journal of Psychiatry, 180 |3|-134.

Mann, D. M. A. (1988) Alzheimer's disease and Down's syndrome. Histopathology, 13, 125-127.

McGuffin, P., Owen, M. J., O’Donovan, M. C., et a (1994) Dementia. In Seminars in Psychiatric Genetics, pp. 192-206. London: Gaskell.

Patel, P., Goldberg, D. \& Moss, S. (1993) Psychiatric morbidity in older people with moderate and severe learning disability. II: The prevalence study. British Journal of Psychiatry, 163, 481-491.

Zigman, W., Schupf, N., Haveman, M., et al (1997) The epidemiology of Alzheimer's disease in mental retardation: results and recommendations from an international conference. Journal of Intellectual Disability Research, 4I, 76-80.

S. Shamas-Ud-Din Arundel House, Smithdown Health Park, Merseycare NHS Trust, Smithdown Road, Liverpool LI5 2LF, UK

Authors' reply: I thank Dr Shamas-Ud-Din for showing interest in my paper and would have to concur with the general criticism that Down's syndrome should have been mentioned. I would like to say, in my defence, that the article was written within the remit of 'advances in old age psychiatry'. A large number of old age psychiatric services see patients with Alzheimer's disease regardless of their age of onset and hence there was a need to cover some of the aspects of the genetics of early-onset Alzheimer's disease. However, I am unaware of any old age psychiatric service within the UK that routinely sees patients with Down's syndrome and Alzheimer's disease. This defence does not, however, excuse a restricted view that is damaging both to patient management and basic research.

Clearly, no patient should be excluded from expert dementia services because of their learning disability. In addition, there is much to learn about the genetic influences on the development of Alzheimer's disease in patients with Down's syndrome and on its clinical phenotype. Thus, as well as the effects of triplication of the amyloid precursor gene, the presence of the APOE $\varepsilon 4$ allele also appears to be associated with an increased risk of developing Alzheimer's disease (Deb et al, 2000). The effect of the presence of the APOE $\varepsilon 4$ allele on age of onset is still unclear but, unlike in those with no learning disability, the presence of APOE $\varepsilon 4$ appears to be associated with an earlier age of death (Hardy et al, 1994). At post-mortem the brain lesions and cholinergic losses seen in individuals with Down's syndrome are the same as those seen in both early- and late-onset Alzheimer's disease. However, despite these neuropathological findings, the evidence for the beneficial effects of cholinesterase inhibitors in patients with Down's syndrome and Alzheimer's disease is still largely anecdotal (Kishani et al, 1999). It is clear that the two specialities, old age psychiatry and learning disabilities, have much to learn from each other.

Deb, S., Braganza, J., Norton, N., et al (2000)

$A P O E \varepsilon 4$ influences the manifestations of Alzheimer's disease in adults with Down's syndrome. British Journal of Psychiatry, 176 468-472.

Hardy, J., Crook, R., Perry, R., et al (1994) Apo E genotype and Down's syndrome. Lancet, 343 979-980.

Kishani, P., Sullivan, J., Walter, B., et al (1999) Cholinergic therapy for Down's syndrome (letter). Lancet, 353, 1064-1065

C. Holmes Memory Assessment and

Research Centre, West Hampshire

NHS Trust, Moorgreen Hospital,

Botley Road,West End, Southampton

SO30 3JB, UK

\section{Clinical diagnosis of dementia with Lewy bodies}

It is clinically important to differentiate dementia with Lewy bodies (DLB) from other types of neurodegenerative dementias because of the prospect of better response to drugs such as cholinesterase inhibitors as well as the risk of development of neuroleptic sensitivity. However, usefulness of the commonly used definition of DLB (McKeith et al, 1996) remains to be established in community and primary care settings. The findings of the Islington study (Stevens et al, 2002) suggest that DLB is a clinically significant type of dementia in the community.

We had an opportunity to look at the prevalence of DLB in a small but representative community sample of patients meeting DSM-IV criteria for dementia (American Psychiatric Association, 1994) in Kerala, a southern state of India. These cases were identified using a novel casefinding method (Shaji et al, 2002). We identified 33 cases of dementia in the study area with a population of 1979 aged $>60$ years. A psychiatrist assessed all the subjects at their residence and assigned a DSM-IV diagnosis. Seventeen people with dementia $(52 \%)$ met criteria for Alzheimer's disease, 12 (36\%) were diagnosed as having vascular dementia, while in $4(12 \%)$ the dementia was felt to be secondary to other conditions such as chronic obstructive pulmonary disease, cardiac failure, Parkinson's disease and subdural haematoma. Three patients (9\%) met criteria for probable DLB and one $(3 \%)$ met criteria for possible DLB when the consensus criteria (McKeith et al, 1996) were applied. The combined prevalence of DLB was therefore $12 \%$. All these patients had earlier met the criteria for Alzheimer's disease as per DSM-IV.

On retrospective application of the separate 'clinical' diagnostic criteria used in the Islington study to our case records, only two of our patients met those criteria, reducing the proportion of patients with DLB to $6 \%$. This is strikingly similar to the figure reported in the Islington study. One limitation of these 'clinical' criteria is that they are too restrictive a definition of DLB. If visual hallucinations are indeed such an important diagnostic marker, then the consensus criteria themselves could be modified by making visual hallucinations an essential symptom for the diagnosis of 